\title{
BMJ Open School-based educational and on-site vaccination intervention among adolescents: study protocol of a cluster randomised controlled trial
}

Paul Gellert, ${ }^{1}$ Norma Bethke, ${ }^{1,2}$ Joachim Seybold ${ }^{2}$

To cite: Gellert P, Bethke N, Seybold J. School-based educational and on-site vaccination intervention among adolescents: study protocol of a cluster randomised controlled trial. BMJ Open 2019;9:e025113. doi:10.1136/ bmjopen-2018-025113

- Prepublication history for this paper is available online. To view these files, please visit the journal online (http://dx.doi. org/10.1136/bmjopen-2018025113).

Received 3 July 2018

Revised 1 November 2018

Accepted 12 December 2018

Check for updates

(c) Author(s) (or their employer(s)) 2019. Re-use permitted under CC BY-NC. No commercial re-use. See rights and permissions. Published by BMJ.

${ }^{1}$ Institute of Medical Sociology and Rehabilitation Science, Charité - Universitätsmedizin Berlin, Berlin, Germany

${ }^{2}$ Medical Directorate, Charité - Universitätsmedizin Berlin, Berlin, Germany

Correspondence to

Norma Bethke;

norma.bethke@charite.de

\section{ABSTRACT}

Introduction Childhood vaccination programmes have been established in all Organisation for Economic Cooperation and Development (OECD) countries; however, measles, mumps and rubella (MMR) as well as diphtheria, tetanus, pertussis and polio (Tdap-IPV) vaccination rates are not optimal in adolescents. Education in combination with easy access vaccination may be a promising approach to improve vaccination rates. We aim at improving MMR and Tdap-IPV rates in a school setting in the context of a planned cluster randomised controlled trial (cRCT), the present paper describes the detailed protocol of this trial.

Methods and analysis We will conduct a schoolbased cRCT, where schools will be randomised to either an educational condition addressing knowledge, risk communication and enhancing self-efficacy regarding vaccination or a low-intensity information condition. In both conditions, a bus equipped with medical staff and materials, will be delivering MMR and Tdap-IPV vaccine directly after the intervention. Schools in the city centre of Berlin, Germany, will be stratified by percentage of migration and type of school. Primary outcome is the number of students who receive vaccination in the bus. Secondary outcomes are knowledge and self-efficacy. An estimated sample size of 355 school classes with approximately 25 students per class is required. The planned analyses will take the nested structure of students, classes and schools into account.

Ethics and dissemination The study will be performed according to the principles of Good Clinical Practice and the Declaration of Helsinki. Approval was obtained by the local ethics committee. Parents of all students will be informed in advance. Their written consent will be obtained, in case students are underage. For dissemination, we will engage with governmental organisations to create potential of our educational unit to be included in future public health prevention schemes. Trial registration number ISRCTN18026662;Pre-results.

\section{INTRODUCTION}

In many countries around the world the vaccination rates for measles, mumps and rubella (MMR) as well as for diphtheria, tetanus, pertussis and polio (Tdap-IPV) are decreasing again. ${ }^{2}$ In multiple European
Strengths and limitations of this study

- Among the first randomised controlled trials targeting vaccinations with a large sample which delivers vaccinations on spot in combination with an educational unit.

- The intervention addressing knowledge, risk communication and enhancing self-efficacy regarding vaccination will be suitable for regular use in schools across students with different socioeconomic and migration backgrounds.

- A limitation concerns the generalisability which is limited to students from schools in the city centre and urban areas.

- With regard to the vaccination rate, one limitation that needs to be addressed is the communication with parents. As an ethical standard, adolescents underage can only get vaccination if the vaccination cards and signed consent declarations by the parents are brought from home.

countries, such as the UK, France, Italy or Spain, several measles outbreaks with more than 1000 cases per year have been observed within the last 10 years. ${ }^{3}$ In 2015, 2465 measles cases were reported in Germany, of which more than $50 \%$ occurred in Berlin. ${ }^{3}$ One reason for this development is the low rate of the second MMR vaccine which is necessary for a lifetime vaccine protection. The most recent vaccination rates at school entrance health examinations in Berlin for the second vaccination were $92.2 \%$ for measles, and 91.8\% for mumps and rubella. ${ }^{1}$ For Berlin adolescents aged 16 years, these rates were even lower at their school entrance examination in 2008 with $88.2 \%$ for measles, and $87.9 \%$ for mumps and rubella. ${ }^{4}$ To prevent the spreading of the virus, more than $95 \%$ population immunity through a two-dose vaccination regimen is warranted. ${ }^{5}$ Effective approaches to increase vaccination rates are needed and school-based interventions may be feasible. 
Although there is no evidence on school-based interventions to increase vaccination rates for MMR or Tdap-IPV, there is a body of literature suggesting the effectiveness of such interventions for other types of vaccinations including sexually transmitted infections/human papillomavirus (HPV) ${ }^{6-11}$ and influenza. ${ }^{12}{ }^{13}$ Frequently, studies applied self-reported outcomes such as knowledge and self-efficacy, ${ }^{7-9} 14$ this draw on theories including Social Cognition Theory ${ }^{15}$ and Protection Motivation Theory. ${ }^{16}$ However, a minority of studies provided the possibility of vaccination directly after the intervention was delivered or report objectively measured vaccination behaviour. ${ }^{6} 1213$

There is mixed evidence about the association of vaccination rates with migration status and socioeconomic background. While studies found lower vaccination rates for those being non-white and below the poverty level ${ }^{17}$ others found associations in the opposite direction. ${ }^{18}$ Research on the effectiveness of school-based MMR or Tdap-IPV vaccination interventions with educational classes in adolescents with varying migration and socioeconomic background is missing.

\section{Trial objectives}

As vaccination rates are decreasing again, our mobile intervention targets an increase in vaccination rates by improving vaccination-related knowledge and self-efficacy in combination with the opportunity of on-spot vaccination. For the main trial of the Prevention Bus project, the primary hypothesis proposes that among adolescents in a school setting, the participation in on-spot vaccination is increased after an educational class compared with a low-intensity information class. In secondary hypotheses, knowledge regarding vaccination and vaccination-related self-efficacy are assumed to be superior in the educational class condition than in the low-intensity class condition after intervention took place. It is assumed that an increase in knowledge and self-efficacy has an influence on the vaccination rate. A spillover effect may become possible if adolescents watch their peers having their vaccination cards checked and being vaccinated. This is discussed in more detail in the context of the theoretical framework. Finally, post-hoc analyses of the intervention effectiveness by gender, migration and socioeconomic background, and type of school will provide further insights about the reach of the intervention for vulnerable subgroups. Using the Prevention Bus as an intervention strategy, barriers will be identified in terms of feasibility to increase the immunisation rates at Berlin schools.

\section{Theoretical framework}

There are various theoretical models of health behaviour change that may be relevant for increasing vaccination rates. The present intervention refers to a framework of constructs from different theoretical models that have effectively been used as a theoretical ground in the development of other vaccination intervention studies. ${ }^{81419}$ It is assumed that vaccine-related self-efficacy and vaccine-related knowledge, as well as a cue to action are associated with an increase in vaccination coverage. ${ }^{20}$

Regarding behaviour change, the Social Cognitive Theory, ${ }^{151}$ which conceptually overlaps with Protection Motivation Theory in the context of vaccination, ${ }^{16}$ is applicable to change of appraisal such as outcome expectancies about vaccination and vaccination-related self-efficacy. This outcome expectancy can be targeted through communication of facts, risks and benefits of vaccinations and infectious diseases. In the same way as knowledge transfer, social factors also are crucial for the development of motivation for a health behaviour like getting a vaccination which can be enabled through observational learning with a fictive or a peer model who decides to have the vaccination card checked or to be vaccinated. Also with group discussions, for example, talking about fears and doubts, ${ }^{22}$ physical and emotional states can be improved and students can verbally persuade each other. Thus, addressing self-efficacy of the students by prompting their mastery experience, the direct possibility to be vaccinated on-site makes the implementation of the behaviour more likely. The Health Belief Model ${ }^{23}$ proposes that threat and perceived benefits (eg, tackled by knowledge provision) and self-efficacy, in conjunction with available cues to action increase the likelihood of engaging in health promoting behaviours such as vaccination. Empirical studies have shown that on-spot vaccination can help to increase the vaccination rate. ${ }^{12} 13$ The possibility of converting intention directly into behaviour after the educational unit, in addition to the promotion of vaccine-related knowledge and enhancing vaccine-related self-efficacy, can lead to a long-term consolidation of behaviour.

\section{METHODS}

The Prevention Bus project is designed to evaluate a school-based vaccination intervention that is mobile and provides low-threshold healthcare provision and health education for young people. The trial was registered previously on ISRCTN and is still ongoing.

\section{Study design}

The planned study design of the main trial is outlined in figure 1 . As preliminary work a pilot study will be carried out, starting 18 September 2017 and running for 4 weeks. The pilot phase was used for testing feasibility of access and recruitment strategies as well as testing measurement instruments and adequacy of our educational material. A publication of the results of the pilot study is planned. The main trial will be running subsequently through the whole winter and summer term until mid of July 2018. At each school we aim to be on-site for 5 days, performing at least three class units per day. With a successful recruitment, including the pilot study, we will spend 34 weeks at various Berlin schools during this period. We will use the prevention bus for this trial, which enables 


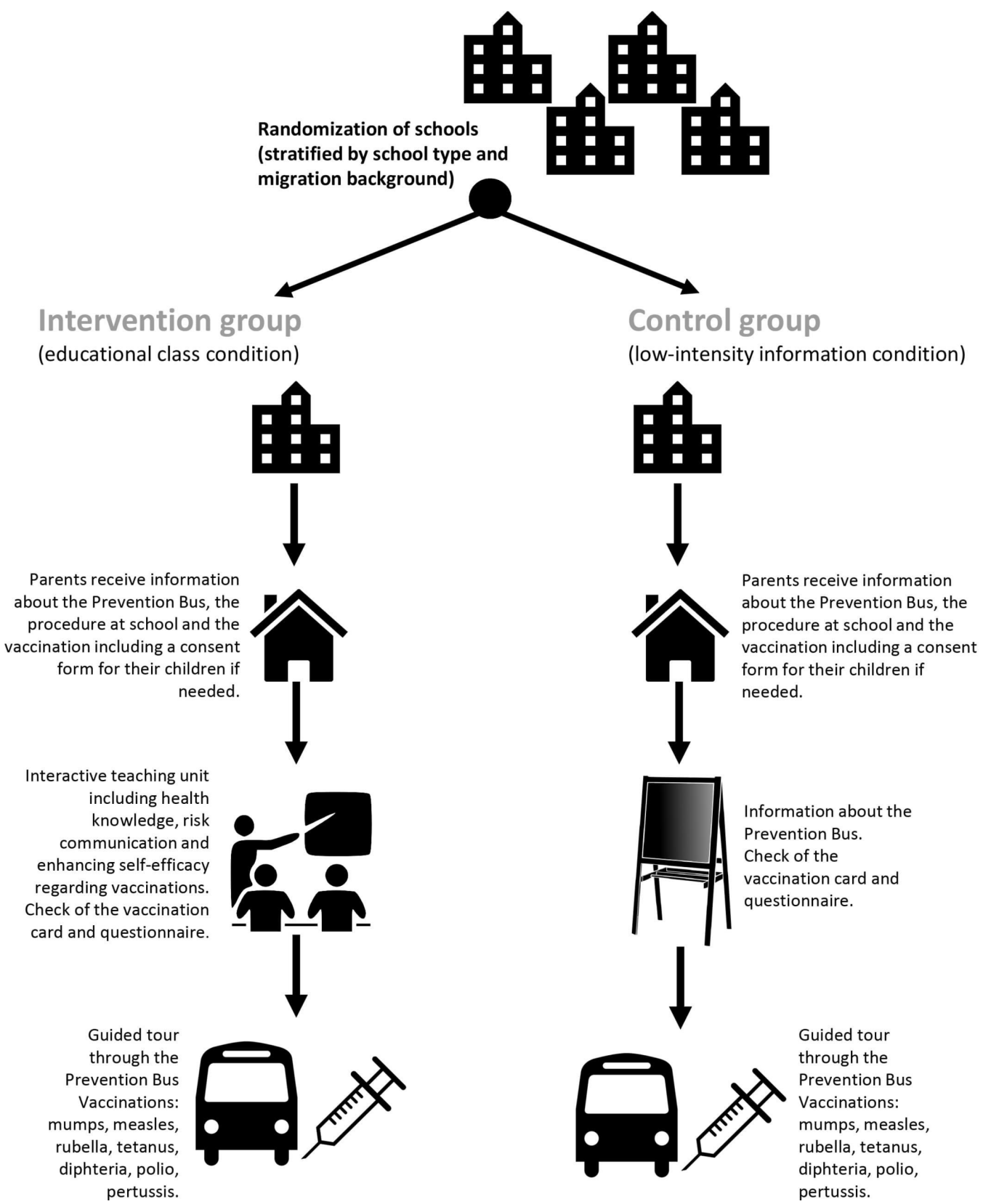

Figure 1 Flow chart of the study design.

mobile medical care of the highest standard. The bus interior was converted by the Deutsche Bahn (German railway company) into a medical practice in 2016 and is equipped with cupboards, water tanks, electricity connections, waiting areas and refrigerators for the vaccines. A separate treatment room with a table, seats and a patient couch is available in the rear part of the bus for the vaccination, which guarantees privacy and the possibility of resting after the vaccination. From the outside the bus is marked red-white and as medibus of the Deutsche Bahn. In addition, the Charité logo will be displayed digitally for our trial.

The planned two-arm cluster randomised controlled trial (cRCT) is going to comprise an educational class condition and a control condition that is a low-intensity information class. Clusters will be randomised at school-level stratified by proportion of students with migration background and school type. In both conditions, parents and students will be informed 1 week in advance about the Prevention Bus, including information about the procedure at school, which will be carried out by nurses and physicians, and vaccines offered. The information about the project will be distributed to students and parents as an official letter with the support of the school director. The schools receive the materials sorted by class and date with preliminary notice by post in advance, on average 1 week ahead. Further, the parents will receive a consent form for vaccination in case students are underage. In order to meet needs of the different linguistic backgrounds of the students' families, the information letter to the study is kept short, being a one pager. In addition to basic information about the study and the 
vaccinations offered, there is also a reference to the possibility of making enquiries on site, by telephone or e-mail. Furthermore, attention is drawn to our homepage, where additional easily understandable material is available for both, parents and students. There they will also find information on vaccination in different languages such as Arabic, Turkish, Russian and Vietnamese, and a declaration of consent that refers to the information provided. ${ }^{24}$ In addition, students with parents who have no or only poor German language skills often play a mediating role in the real-life school-home context. Since we use the same way of communication, via the school management, we assume that students communicate as usual in such situations with their parents. The extent to which the migration background has an influence or not on the response to the declarations of consent will be examined in the secondary analyses of the data. When the bus is on-site at schools all students, including those underage, will be asked for their consent right before vaccination takes place. In Germany, parents' consent to the vaccination of students under the age of 18 is considered as a necessary condition for the vaccination of underaged. However, the additional consent on-site to vaccinate given by the adolescents themselves is regarded as a sufficient condition to carry out an actual vaccination. If underage students decide to be vaccinated but their parents have not yet given their consent, they have the opportunity to get a new consent form from the bus team on-site and present it to their parents at home and to receive vaccinations on one of the following days, while the bus is still at their school.

At school-level, schools with upper secondary education/vocational schools will be eligible to be included in the trial if they are located in a borough of the city centre of Berlin, Germany. All boroughs, which are at least partly within the Berlin circle line, are defined as city centre. Circle line serves as a definition criterion for the centre of Berlin, as it provides a clear distinction between central and suburb area of Berlin. In addition, the time should be used to the maximum and the bus, which depot is located in a central borough, should have time-efficient routes. Schools with less than 200 students in the classes from 9th to 11th grade in upper secondary schools or for all apprentices in their teaching facilities are not eligible. Originally, all schools with fewer than 230 students were to be excluded, but as classes were smaller than expected, this led to an adjustment of the minimum number. At class level, all classes participating in regular classes, meeting the criteria listed above, are included in the study. However, in some schools in Berlin in this age group, there are so-called 'welcome classes' (Willkommensklassen). These adolescents have limited knowledge of German, because they have not yet lived in Germany for long since they recently emigrated from their homeland. In these classes, a vaccination card control is offered if required and teaching in simplified German is carried out if time resources are available. Nonetheless, these classes will not be part of the sample. On an individual-level, students will be included when aged minimum 15 years and for on-spot vaccination additionally a signed consent form is required, when students are underage, it needs to be signed additionally by the parents.

When schools are contacted, the recruiting person will be blinded to the intervention allocation of the school, as the intervention condition is blacked out on the school list. The recruiting person therefore has no knowledge of the assignment during recruitment. For each cluster (eg, high school with high migration status), the same number of schools are first contacted by postal mail and asked to participate. In addition to the personal cover letter, there will be an information flyer. Within this first contact, the general project is described and that $45-90 \mathrm{~min}$ are required per class. If no feedback is received, the school will be reminded of the project by email once more with information material. In further absence of feedback, a telephone call and/or a personal appointment are then scheduled. If a school cancels despite all activities, another school within the same cluster will be contacted. The cluster lists all available schools, all of which were previously assigned randomly to the intervention conditions.

\section{Intervention and control condition}

When the Prevention Bus is at a school, all students will be given the possibility to get information and vaccination if needed. Besides that, there will be the intervention taking part in the classes from 9th to 11th grade in high schools and for all apprentices in their teaching facilities. Either the class will be an educational class condition or a low-intensity information condition based on the randomisation. Trained physicians and nurses will deliver the intervention at schools. For organisational reasons, they will know to which intervention condition the school belongs, from the moment they arrive at the school. However, in advance neither the school staff nor the students will be aware of the details about the other condition of intervention. There will be a debriefing at the end of the school year. All headmasters will be informed by mail about the level of intensity of the information given in the class. Via the headmasters, the information is to be passed on to the respective class teachers and students, who have participated in the trial.

The educational class condition is scheduled with $90 \mathrm{~min}$ and the low-intensity information condition with $45 \mathrm{~min}$. Both units for each class will begin in the classroom but differ in the amount and format of information provided. Both groups in the beginning will receive basic information on the Prevention Bus and vaccinations offered and that they can be vaccinated directly afterwards in the bus, if a vaccination is outstanding, they decide positively for a vaccination and they have the signed parent consent thereby. The educational class condition will focus on knowledge, risk communication and enhancing self-efficacy regarding vaccination. The low-intensity information condition will only give basic information. Detailed differences are described below. Both conditions include an anonymous questionnaire on sociodemographic 
background, self-efficacy and knowledge regarding vaccination, which is delivered in the classroom, before students go to the bus. Furthermore, the check of the vaccination card in the classroom and a guided tour through the Prevention Bus, led by two nurses, will be part of the intervention for both groups. In both cases, the language of instruction will be German. One focus of the study is on the migration background of adolescents which in some schools can be very high. However, the intervention takes place under real conditions and students in all participating classes also take part in regular German-language lessons in everyday school life. Nevertheless, the training of the physicians was designed in advance to adapt to different contexts. Following the class, the physicians will fill out a short quality assurance questionnaire which will allow double checks to study data (teaching quality, problems, number of students, number of vaccination cards). After the bus tour, all students with an indication and with a signed consent form, by themselves and additionally by their parents if student is underage, can be vaccinated. Even though underage students need parental consent for vaccination, the final decision to be vaccinated is up to the students themselves. For ethical demands, no link may be established between the questionnaire data from the class and the individual data after vaccination. On an anonymous level, the class and the sociodemographic background are again collected from the vaccinee. Demands will be elaborated further in the Ethics approval and consent to participate section. All students who receive vaccinations are vaccinated in the separate treatment room at the rear of the bus. Students do not watch each other getting vaccinated. However, their peers will accompany them when they walk from the classroom to the bus which may increase social commitment, but guarantees privacy at the same time. If desired, adolescents may be accompanied by their peers during vaccination. Since the bus is always on-site for several days, students without signed parental consent and/or vaccination card are always offered to have their vaccination card checked on the bus in the following days and to be vaccinated if necessary. This is done regardless of the condition of the intervention. If the students are underage, the bus team will also give them a consent form to take home for the parents. In this way, students can discuss with their parents the vaccination decision in more detail. In order to assess lagged decisions, additional documentation will be provided. If students are subsequently on the bus for the check of the vaccination card and/or vaccination, the date (distance to intervention), their class and the questionnaire on sociodemographic background are documented. This corresponds to the same procedure for students who are vaccinated immediately after the educational unit.

\section{Content of conditions}

The educational class consists of a digital Powerpoint presentation held by a physician, including photo and video material which allows standardised information delivery. In addition to the standardised digital presentation, all intervention facilitators will obtain a script for the presentation, and they will be training the presentation in front of the study team in advance including frequent feedback of experienced colleagues before and during the trial. The amount of information provided is intended to be kept at a low level but still capturing all relevant aspects. In addition, presentation of content will be fit to the target group as described further on (eg, wording, use of photo and video material, activating elements). Informed by Social Cognition and Protection Motivation Theory, ${ }^{1516}$ the content of the class will be targeting three mechanisms, that is, increasing knowledge by provision and questioning of information, ${ }^{25}$ risk perception by risk communication, ${ }^{26}$ enhancing self-efficacy ${ }^{27}$ by a testimonial and a guided group discussion. Information on the basics of the immune system and the vaccination process will be provided in order to understand how vaccines work. Throughout the class, knowledge of the students will be questioned and corrected if necessary. In addition, background information will be given on all seven infectious diseases against which vaccinations are offered on spot at the bus. Further, information on social aspects will be given, addressing herd and nest immunity (ie, vaccinations protect not only oneself but also other people.). The exchange of different perspectives and experiences considers also emotional dimensions, important for lasting learning. All physicians, who will conduct the educational unit, are trained to adopt a cooperative teaching format. ${ }^{28-30}$ Provision of information and questioning of knowledge was chosen as an interactive and constructive method of connecting new information with prior knowledge. This also includes frequent feedback rounds conducted by research associates involved in the development of the educational class. Adequacy and feasibility of the educational class unit will be tested in the piloting phase. Changes will be made for the main trial if necessary.

Risks and advantages of vaccinations are communicated in detail on measles. Adapted to the target group, chances will be communicated in whole numbers supported by graphical depictions (eg, 93 out of 100 students get infected with measles if they are not vaccinated). In order to enhance self-efficacy regarding vaccination, a group discussion is initiated by the physician after the basics of the vaccination process are implemented. Triggered by a fictive social media post of a fictive person (ie, testimonial/role model), with a gender-neutral name and look, students have the opportunity to exchange with each other about negative apprehensions, doubts and questions they have on vaccinations. In the end of the class, the ideas such as social aspects and risk-associated knowledge will be taken up again and will be reflected. The questionnaire will be filled out after the class has finished. The intervention will end with a guided tour through the Prevention Bus, where vaccination will take place.

The low-intensity information group will get a brief introduction to the project and the Prevention Bus ('Why 
are we here') lasting about 5 min. No more information is provided. Identically to the educational class group, the questionnaire will be filled out in the classroom. Subsequently, the class gets a guided tour through the bus, where also vaccinations are offered.

Parents in both groups will be informed in an identical manner about the project and vaccinations offered at least 1 week prior to the intervention. Apart from general information about the project, the announcement will include a declaration of informed consent to allow vaccination of their children, when underage, as well as detailed health information regarding the diseases and vaccinations. All parents do have the opportunity to get more information on the project website and via phone or email which will be attended by a study assistant.

\section{Outcome assessment}

The primary outcome will be composite MMR and Tdap-IPV vaccination rate. Vaccination rate is objectively measured through the number of students who get vaccinated, directly after receiving treatment (educational class condition, low-intensity information condition) when students visit the Prevention Bus and those students who get vaccinated subsequently in the following days, when the bus is still on-site. The primary outcome will be weighted by the number of students who are eligible to take part in the study.

Secondary outcome are health knowledge and self-efficacy. Following outcome-based teaching theory (ie, constructive alignment of the content of teaching with test items $^{31}$ ), health knowledge will be measured with six immunisation knowledge items, reflecting the main topics of the intervention.

Self-efficacy concerning the competency to understand and appraise vaccination related health information is measured using a five items short-version of the European Health Literacy Survey Questionnaire 47, where we selected those items, which were addressing prevention and immunisation treatment. ${ }^{32} 33$

The questionnaire will be administered before students visit the bus while they are still in their classroom. The data entry will be carried out by the bus team (nurses, physicians) directly after the intervention has taken place.

Additionally, potential confounders including gender, socioeconomic and migration status, and vaccination status will be assessed (ie, objective vaccination status prior to the intervention taken from students' vaccination cards). In addition to mumps, measles, rubella, tetanus, diphtheria, pertussis and polio, the vaccination status of all other vaccinations recommended by the Permanent Vaccination Committee of the German government's central scientific institution the Robert Koch-Institute will be documented.

\section{Randomisation}

In a first step, all eligible schools in Berlin are contacted via the head of school following a stratified random order until 30 schools will be confirming participation. Strata will be (a) percentage of adolescents with migration background (below vs above median) and (b) type of school (high school, vocational school, integrated secondary school) using RAND function in Microsoft Excel 2013. Further, a stratified school-level cluster block randomisation to either educational class or low-intensity information condition with strata using blockrand package in $\mathrm{R}$ V.3.2.1 was performed.

\section{Estimated sample size}

Within this trial, in all, 30 schools (high school, vocational school, integrated secondary school) will be approached. At each school, the team of the prevention bus aims to be on-site for 5 days and will address at least three school classes per day. This results in an anticipated sample size of 510 school classes for the entire survey period. In the city centre of Berlin, there are a total of 19 vocational schools, 15 integrated secondary schools and 41 high schools which are potential candidates for our model project.

In correspondence with our intervention procedure and to avoid contamination, the unit of randomisation was school. This will result in establishing a cRCT design where units (ie, classes) were randomised together in clusters (ie, schools). We expect potential contamination effects to be moderate within classes (ie, across students within one class), but smaller across classes within one school. ${ }^{8}$ Further, the education was delivered at class level. Thus, we decided the unit of sample size estimation will be class.

In the planned cRCT, stratified by school type and migration rate, the schools are randomly assigned with a 1:1 ratio to either the educational condition or low-intensity information condition. In order to achieve an adequate vaccination coverage of $97 \%$ in the primary endpoint (MMR, Tdap-IPV vaccination) on a class-level, a required sample size of 355 school classes was determined a-priori (z-test; $88 \%$ MMR initial vaccine rate and a difference to the control group of $7 \%$ ). The estimation is based on an intraclass coefficient of $0.02,{ }^{13}$ a one-sided alphalevel of 0.05 and a statistical power of 0.8 using GPower V.3.1.9.2. ${ }^{34}$

\section{Risk assessment}

Risks and disadvantages are not to be expected from the intervention. For MMR and Tdap-IPV vaccinations, vaccination side effects are expected to be minimal, and serious side effects are expected to be extremely rare..$^{35} 36$ The likelihood of occurrence of vaccination complications (eg, transient therapies-related disease) is in the range of 1 per $1000 .{ }^{37}$ However, detailed information and medical history takes place before vaccination. According to the recommendations of the Permanent Vaccination Committee of the German government's central scientific institution the Robert Koch-Institute, before the administration of vaccination, detailed information on vaccination and vaccination complications must be provided. ${ }^{24}$ 
As part of our study quality assurance, negative events will be recorded and reported.

\section{Ethics approval and consent to participate}

An information letter about the project for parents and children and a letter with detailed information on MMR and Tdap-IPV vaccination asking for consent to participate will be sent to the school 1 week before the project was implemented in respective schools. Schools distributed the information material to every class through class teachers. Thereby, the study team would not be contacting the parents directly. To protect the personal information and rights of the students, the ethics committee has mandated that questionnaire data from the classroom should not be linked to vaccination data from the prevention bus which will allow us to carry out certain analyses only at the class level.

\section{Analysis plan}

Primary and secondary outcomes by intervention allocation will be analysed applying Generalized Estimation Equations adjusting for the nested structure (ie, school, classes, students) to regress individual-level on-site vaccination rate/knowledge/self-efficacy on intervention condition when accounting for individual-level type of vaccination received, class-level socioeconomic and migration status, age and school-level type of school using GENLIN procedure in SPSS V.25. Results will be reported by the unit of randomisation (ie, by schools) and as overall estimated averages for intervention and control condition. Missing data $>5 \%$ will be handled using multiple imputation. Post-hoc analyses, which do not match class room questionnaire data with on-site vaccination data, but refer to just one of these, will be possible on an individual level of analysis and will be analysed using mixed modelling accounting for clustering of students within classes within schools.

\section{Patient and public involvement}

Intensive dialogues with political members of the German Federal Ministry of Health drove the development of the research question, and we have been discussing the relevance and the design of the study with the headmasters of schools. Subsequently, students are involved in the design of this study as part of the piloting as described. Further, within the pilot phase, we will be testing all outcome measures which will be adjusted according to the feedback of the students. The students' priorities and preferences were taken into account by extent and format of information presented. However, students will not be involved in the recruitment and conduct of the study. After the main trial, all students will be informed via the headmasters and the respective teachers by information provision on details of the educational unit as well as on the background of the study and results regarding vaccination rate, and related knowledge and self-efficacy regarding vaccination. Further, we will allow all students to contact us via mail for additional questions or doubts.
Finally, although we do not include a formal assessment of participant burden, students within the pilot and the main phase will be invited to provide feedback about positive and challenging issues with a short online questionnaire provided to the headmasters of their schools. For further dissemination of the educational unit, beyond this trial, we will be in contact with governmental institutions including the German Federal Ministry of Health. We aim to ensure that the educational unit will inform the national prevention strategy. ${ }^{38}$

\section{DISCUSSION}

The objective of this article was to describe our planned school-based educational and on-site MMR and Tdap-IPV vaccination intervention. The findings of our cRCT will provide evidence on the accessibility of adolescents to mobile on-site healthcare services as well as on their vaccination uptake. In the context of the Social Cognitive Theory, our educational intervention targeting three mechanisms, that is, increasing knowledge ${ }^{25}$ and risk perception, ${ }^{26}$ and enhancing self-efficacy. ${ }^{27}$ These mechanisms are expected to work in combination with an on-site MMR and Tdap-IPV vaccination opportunity directly after the educational class took place.

By means of our trial, results might lead to an increase in the vaccination rate which is of great public health relevance. By June 2018, again more than 200 measles cases had already been reported in Germany. ${ }^{3}$ The national vaccination target of removing measles in Germany by $2020^{38}$ is a long way off and intervention projects like ours are urgently needed. Further, for the goal of broad acceptance of vaccinations of the $\mathrm{WHO},{ }^{39}$ our proposed intervention appears to be an appropriate tool to reach adolescents at scale.

\section{Strengths and limitations}

Our study will be among the first randomised controlled trials targeting vaccinations with a large sample which delivers vaccinations on spot in combination with an educational unit suitable for regular use in schools. The mobile approach enables an extremely efficient, comprehensive supply of vaccinations. Moreover, we address adolescents who, because of their age, soon become multipliers in their professional or family environment.

Our study has several limitations worth noting. With regard to the vaccination rate, one limitation that needs to be addressed is the communication with parents. As an ethical standard, the vaccination rate can only be collected and, if necessary, adolescents vaccinated if the vaccination cards and consent declarations are brought from home. A greater focus on methods of communication is likely to be relevant for future studies. Due to time constrictions, we will administer the questionnaire in the educational class unit only after the class is held. Although baseline assessments allow for controlling for baseline differences across groups, in RCT designs, differences 
are controlled by the randomisation procedure and are purely by chance. ${ }^{40}$

\section{Future research}

In addition to MMR and Tdap-IPV vaccinations, future studies should address HPV vaccinations, as this is still a relatively recent vaccination. The mobile approach of carrying out vaccination programmes supported by a medical team and a bus with vaccinations offered on spot seem to be a fruitful public health strategy. This especially holds true for urban spaces like Berlin, where vaccination rates are far from being optimal. ${ }^{4}$ Policy has to deal with how such offers can be meaningfully integrated into their political agenda and subsequently into healthcare practice. While local physicians are supported by such an approach; likewise, it would be possible to include them in the design of mobile vaccination strategies. Further, possibilities of mobile medical strategies for medically underserved regions lacking preventive health such as certain rural areas should be developed and evaluated in order to improve their healthcare quality. Along these lines, synergy can be created between public health strategies and day-to-day medical care.

\section{Conclusion}

The innovative approach of providing education and vaccinations with a bus by mobile means enables attractive routes for medicine and politics to provide comprehensive preventive medical care. Given this background, the use and dissemination of evidence-based educational materials to the public is extremely valuable. In the future, we will be confronted with ever more structural changes in the population in urban and rural areas, where mobile healthcare services carry an opportunity to optimise healthcare delivery cooperatively and evidence based interventions are urgently needed.

Acknowledgements The authors would like to thank the entire bus team, Charité - Universitätsmedizin Berlin, including physicians, nurses and our bus driver for their positive influence on the project during the planning and implementing phase. We would also like to thank our team in the office for their support in planning and recruiting. Furthermore, we would like to thank Professor Nina Knoll for her valuable comments on our manuscript. Additionally, we acknowledge support from the German Research Foundation (DFG) and the Open Access Publication Fund of Charité - Universitätsmedizin Berlin.

Contributors The trial organisation, trial management and data monitoring will be carried out by the study team (NB, JS). The study is designed and planned by the Medical Directorate, Charité -Universitätsmedizin Berlin (NB, JS) and methodological support is provided by the Medical Sociology, Charité Universitätsmedizin Berlin (PG). NB, PG, JS will have access to the final trial dataset. NB and JS will be responsible for requests regarding the anonymised dataset. The study evaluation will be separately conducted by PG and NB. The random sequence allocation was carried out by PG. Participant enrolment was carried out by NB. The teaching unit is developed by NB with advice from PG and JS.

Funding The study was funded by the German Federal Ministry of Health (BMG; Grant number 1503/53105). The study design was developed and will be carried out as well as the data monitoring independently from the study sponsor. Address: Friedrichstraße 108, 10117 Berlin, Germany.

Competing interests None declared.

Patient consent for publication Not required.
Ethics approval The procedure of the main trial was approved by the Ethics Committee of the Charite - Universitätsmedizin Berlin (EA1/059/17). All students, including those underage, will be asked for their consent right before vaccination takes place. Parental consent will be obtained also in advance, in case students are underage.

Provenance and peer review Not commissioned; externally peer reviewed.

Open access This is an open access article distributed in accordance with the Creative Commons Attribution Non Commercial (CC BY-NC 4.0) license, which permits others to distribute, remix, adapt, build upon this work non-commercially, and license their derivative works on different terms, provided the original work is properly cited, appropriate credit is given, any changes made indicated, and the use is non-commercial. See: http://creativecommons.org/licenses/by-nc/4.0/.

\section{REFERENCES}

1. Robert Koch-Institut (RKI). [Vaccination rates in Germany 2015] Impfquoten bei der Schuleingangsuntersuchung in Deutschland 2015. Epidemiologisches Bulletin 2017;16:137-48.

2. UNICEF. Immunization coverage and system performance indicator data: UNICEF, 2018.

3. World Health Organization (WHO). Measles and Rubella Surveillance data. Geneva, Switzerland, 2017.

4. Robert Koch-Institut (RKI). [Vaccination rates in Germany 2008] Impfquoten bei der Schuleingangsuntersuchung in Deutschland 2008. Epidemiologisches Bulletin 2010;16:137-46.

5. Andre FE, Booy R, Bock HL, et al. Vaccination greatly reduces disease, disability, death and inequity worldwide. Bull World Health Organ 2008;86:140-6.

6. Rickert VI, Auslander BA, Cox DS, et al. School-based HPV immunization of young adolescents: effects of two brief health interventions. Hum Vaccin Immunother 2015;11:315-21.

7. Kwan TT, Tam KF, Lee PW, et al. The effect of school-based cervical cancer education on perceptions towards human papillomavirus vaccination among Hong Kong Chinese adolescent girls. Patient Educ Couns 2011;84:118-22.

8. Grandahl M, Rosenblad A, Stenhammar C, et al. School-based intervention for the prevention of HPV among adolescents: a cluster randomised controlled study. BMJ Open 2016;6:e009875.

9. Coyle KK, Glassman JR, Franks HM, et al. Interventions to reduce sexual risk behaviors among youth in alternative schools: a randomized controlled trial. J Adolesc Health 2013;53:68-78.

10. Cox DS, Cox AD, Sturm L, et al. Behavioral interventions to increase $\mathrm{HPV}$ vaccination acceptability among mothers of young girls. Health Psychol 2010;29:29-39.

11. Forster AS, Cornelius V, Rockliffe L, et al. A cluster randomised feasibility study of an adolescent incentive intervention to increase uptake of HPV vaccination. Br J Cancer 2017;117:1121-7.

12. Daley MF, Kempe A, Pyrzanowski J, et al. School-located vaccination of adolescents with insurance billing: cost, reimbursement, and vaccination outcomes. J Adolesc Health 2014;54:282-8.

13. Humiston SG, Schaffer SJ, Szilagyi PG, et al. Seasonal influenza vaccination at school: a randomized controlled trial. Am J Prev Med 2014;46:1-9.

14. Gottvall M, Tydén T, Höglund AT, et al. Knowledge of human papillomavirus among high school students can be increased by an educational intervention. Int J STD AIDS 2010;21:558-62.

15. Bandura A. Social cognitive theory: an agentic perspective. Annu Rev Psychol 2001;52:1-26.

16. Rogers RW. A protection motivation theory of fear appeals and attitude change1. J Psychol 1975;91:93-114.

17. Centers for Disease Control and Prevention (CDC). National, state, and local area vaccination coverage among adolescents aged 13-17 years-United States, 2009. MMWR Morb Mortal Wkly Rep 2010;59:1018-23.

18. Walter D, Atzpodien K, Pins C, et al. [Factors influencing the uptake of vaccines by adolescents with migration background. A qualitative study of adolescents, mothers, and physicians] Einflussfaktoren auf die Inanspruchnahme von Impfungen durch Jugendliche mit Migrationshintergrund. Bundesgesundheitsblatt Gesundheitsforschung Gesundheitsschutz 2013;56:1368-75.

19. Davies C, Skinner SR, Stoney T, et al. 'Is it like one of those infectious kind of things?' The importance of educating young people about HPV and HPV vaccination at school. Sex Educ 2017;17:256-75.

20. Brewer NT, Chapman GB, Rothman AJ, et al. Increasing vaccination: putting psychological science into action. Psychol Sci Public Interest 2017;18:149-207. 
21. Bandura A. Self-efficacy mechanism in human agency. Am Psychol 1982;37:122-47.

22. Bandura A. Health promotion by social cognitive means. Health Educ Behav 2004;31:143-64.

23. Becker $\mathrm{MH}$. The health belief model and personal health behavior. Health Education Monographs 1974;2:324-473.

24. Robert Koch-Institut (RKI). [Information material on vaccination in different languages] Informationsmaterialien zum Impfen in verschiedenen Sprachen. 2018 https://www.rki.de/DE/Content/ Infekt/Impfen/Materialien/materialien_fremdsprachig_inhalt.html

25. Wegwarth O, Kurzenhäuser-Carstens S, Gigerenzer G. Overcoming the knowledge-behavior gap: The effect of evidence-based HPV vaccination leaflets on understanding, intention, and actual vaccination decision. Vaccine 2014;32:1388-93.

26. Galesic M, Garcia-Retamero R, Gigerenzer G. Using icon arrays to communicate medical risks: overcoming low numeracy. Health Psychol 2009;28:210-6.

27. Usher EL, Pajares F. Sources of self-efficacy in school: critical review of the literature and future directions. Rev Educ Res 2008;78:751-96.

28. Wyatt KD, List B, Brinkman WB, et al. Shared decision making in pediatrics: a systematic review and meta-analysis. Acad Pediatr 2015;15:573-83.

29. Katz AL, Webb SA. Committee on bioethics. Informed consent in decision-making in pediatric practice. Pediatrics 2016;138:e20161485.

30. Lipstein EA, Brinkman WB, Fiks AG, et al. An emerging field of research: challenges in pediatric decision making. Med Decis Making 2015;35:403-8.

31. Yusof KM, Hassan SAHS, Phang FA. Creating a Constructively Aligned Learning Environment using Cooperative Problem Based Learning (CPBL) for a Typical Course. Procedia Soc Behav Sci 2012;56:747-57.
32. Röthlin F, Pelikan JM, Ganahl K. Health Literacy of 15 year old adolescents in Austria. Final report of the Austrian Health Literacy youth study] Die Gesundheitskompetenz der 15-jährigen Jugendlichen in Österreich. Abschlussbericht der österreichischen Gesundheitskompetenz Jugendstudie im Auftrag des Hauptverbandes der österreichischen Sozialversicherungsträger (HVSV). Wien: Ludwig Boltzmann Institut Health Promotion Research, 2013.

33. Sørensen K, Van den Broucke S, Pelikan JM, et al. Measuring health literacy in populations: illuminating the design and development process of the European Health Literacy Survey Questionnaire (HLSEU-Q). BMC Public Health 2013;13:948.

34. Faul F, Erdfelder E, Lang A-G, et al. G*Power 3: a flexible statistical power analysis program for the social, behavioral, and biomedical sciences. Behav Res Methods 2007;39:175-91.

35. Demicheli V, Rivetti A, Debalini MG, et al. Vaccines for measles, mumps and rubella in children. The Cochrane database of systematic reviews 2013;2:Cd004407.

36. Farez MF, Correale J. Immunizations and risk of multiple sclerosis: systematic review and meta-analysis. J Neurol 2011;258:1197-206.

37. Barlow WE, Davis RL, Glasser JW, et al. The risk of seizures after receipt of whole-cell pertussis or measles, mumps, and rubella vaccine. N Engl J Med Overseas Ed 2001;345:656-61.

38. Aktionsplan AG. [National Action Plan 2015-2020 on the elimination of measles and rubella in Germany] Nationaler Aktionsplan 20152010 zur Elimination der Masern und Röteln in Deutschland. 2015 https://wwwgmkonlinede/documents/Aktionsplan_Masern_ Roeteln_2pdf.

39. World Health Organization (WHO). Global measles and rubella strategic plan: 2012-2020. Geneva, Switzerland, 2012.

40. Roberts $\mathrm{C}$, Torgerson DJ. Baseline imbalance in randomised controlled trials. BMJ 1999;319:185. 